adults, but are rarely seen as optimum sites for student learning, or as vibrant spaces for personal and professional growth for staff or residents. Creating a supported learning environment within these care settings is vital to enhance quality of care and quality of life for residents, their families and staff, and to promote effective learning experiences for students.

The Faculty of Nursing at the University of Calgary is working with Covenant Care, a non-profit care provider organization, on an innovative partnership aimed at developing a learning culture within a complex care environment in Calgary, Alberta. This enhanced learning partnership is comprised of three core elements: 1) an undergraduate nursing positive placement program $(+\mathrm{PPP}) ; 2$ ) research and advanced practice learning opportunities for graduate students; and 3) learning opportunities and workforce development for residents, families and staff. This participatory action research study is exploring and developing a personcentered care and learning culture within a supportive living and hospice setting.

This presentation will detail our developing understanding of the core components of a learning culture within long-term care settings, as well as aspects of culture change, including barriers and facilitators, culture change process, and challenges associated with evaluation and sustainability.

\section{ALIGNING TEACHING IN GERIATRICS WITH CORE ENTRUSTABLE PROFESSIONAL ACTIVITIES FOR ENTERING RESIDENCY}

H.Y. Cheng, M. Gusic, Medicine, UVA, CHARLOTTESVILLE, Virginia

The Core Entrustable Professional Activities (EPAs) for Entering Residency ${ }^{1}$ define behaviors that medical students should be able to do to ensure readiness for residency. Each is mapped to the Physician Competency Reference Set ${ }^{2}$ but mapping to the Minimum Geriatrics Competencies (MGC) for Graduating Medical Students ${ }^{3}$ has not been reported.

Based on MGC, the author created workshops on 1) identifying and reducing hidden harms of drug therapy, 2) practicing value- and preference-based prescribing/deprescribing, 3 ) prescribing opioids and 4) writing admission orders. These workshops address the competencies critical to entrustment for EPA 4 through addressing key functions ${ }^{1}$ associated with the task.

The following illustrates how teaching and assessment of MGC can be used to meet curricular goals and provide assessment data about student performance of EPAs. A workshop designed to facilitate students' ability to "accurately identify clinical situations where...patient preference, or goals of care should override standard recommendations for treatment in older adults" (MGC 16) addresses the key function, "demonstrate an understanding of the patient's current condition and preferences that will underpin the orders being provided" and critical competency, "make informed decisions about diagnostic and therapeutic interventions based on patient information and preferences, up-to-date scientific evidence, and clinical judgment" linked to EPA 4 (ability to enter and discuss orders and prescriptions). Assessment of student performance is done through pre- and post-tests and clinical observations. Data from the assessments are used in the summative evaluation for the clerkship.

\section{LIVE-IN STRANGERS: AN EXPERIENTIAL ACCOUNT OF GERONTOLOGY EDUCATIONAL IMMERSION IN SENIOR HOUSING}

R. Kilaberia, E. Ratner, School of Social Work, University of

Minnesota, Minneapolis, Minnesota

Gerontology education programs that combine elements of experiential service-learning, community participation, and residential immersion have been emerging in the United States and internationally. Combining fieldwork, interviews, and immersion methods, this study draws on qualitative ethnographic and phenomenologic design, whereby a student lived in senior housing for 3.5 years. The study is among the longest and most intensive gerontological field experiences ever reported. It is based on more than 2,000 hours of observations and conversational interviews with 14 residents. Major patterns based on observational data show the importance of shared history, support for one another, negotiating environmental tensions, and demand for attention on the part of residents. Major themes derived from interviews elaborate on family conflict, conflict between residents, and life decisions. Results show that a unique combination of physical and social proximity in an intergenerational alliance enables both life enhancement for older adults and learning opportunities for students. A recommendation is made to implement a live-in role more formally.

\section{AN INTERPROFESSIONAL GERIATRIC TRAINING AND OUTREACH PROGRAM: STUDENT, PATIENT, AND PROVIDER OUTCOMES}

J.M. Shellman ${ }^{1}$, M. Malcolm ${ }^{1}$, A. LeClaire ${ }^{1}$, J. Cave ${ }^{1}$, C. Rees ${ }^{2}$, 1. University of Connecticut, Storrs, Connecticut, 2. Middlesex Hospital, Middletown, Connecticut

The need for older adults to access high quality care can only be fully addressed with a workforce well-trained in the specialty of geriatrics. Geriatric Outreach and Training with Care (Got Care!) is an innovative, interprofessional geriatric training and practice model designed to develop a cadre of healthcare providers skilled in interprofessional, geriatric care. This academic-community partnership includes an interdisciplinary faculty team of geriatric experts from nursing, medicine, dental medicine, pharmacy, physical therapy, social work, and public health. Unique hands-on opportunities are provided for interprofessional students to learn together in the didactic arena and then reach out with geriatric expert faculty to conduct interprofessional home visits to older adults with multiple chronic conditions and high emergency department (ED) use. The purpose of this study was to evaluate the impact of the GOT! Care Program on student ( $\mathrm{N}=177$ ) collaborative practice skills, patient outcomes $(\mathrm{N}=38)$, and primary care provider $(\mathrm{N}=12)$ satisfaction. Statistically significant improvement was found in student levels of collaborative practice skills in 7 of 8 subscales of the Collaborative Practice Assessment Tool. Improvements in patient experience with care were noted including increases in quality and satisfaction as measured by HEDIS, an increase in referrals to community services such as mental health, social work, and palliative care services, and a decrease in ED use in the sample. Primary care providers report high satisfaction with the program citing GOT! Care, "a more cohesive approach" 